\title{
Clinical evidence of the nonpathogenic nature of the M34T variant in the connexin 26 gene
}

Delphine Feldmann ${ }^{1}$, Françoise Denoyelle ${ }^{2}$, Natalie Loundon ${ }^{2}$, Dominique Weil ${ }^{3}$, Erea-Noel Garabedian ${ }^{2}$, Remy Couderc ${ }^{1}$, Alain Joannard ${ }^{4}$, Sébastien Schmerber ${ }^{5}$, Bruno Delobel $^{6}$, Jacques Leman ${ }^{7}$, Hubert Journel ${ }^{8}$, Hélène Catros ${ }^{9}$, Claude Ferrec ${ }^{10}$, Valérie Drouin-Garraud ${ }^{11}$, Marie-Françoise Obstoy ${ }^{12}$, Lucien Moati ${ }^{2}$, Christine Petit ${ }^{3}$ and Sandrine Marlin*,13

${ }^{1}$ Service de Biochimie et de Biologie Moléculaire, Hôpital d'Enfants Armand-Trousseau, AP-HP, Paris, France; ${ }^{2}$ Service d'ORL et de Chirurgie Cervico-Faciale, Hôpital d'Enfants Armand-Trousseau, AP-HP, Paris, France; ${ }^{3}$ Unité de Génétique des Déficits Sensoriels, Institut Pasteur, Paris, France; ${ }^{4}$ Service de Pédiatrie, CHU, Grenoble, France; ${ }^{5}$ Service $d^{\prime} \mathrm{ORL}, \mathrm{CHU}$, Grenoble, France; ${ }^{6}$ Centre de génétique, Hôpital St Antoine, Lille, France; ${ }^{7}$ Centre Rochin, Lille, France; ${ }^{8}$ Unité de génétique médicale, CHR, Vannes, France; ${ }^{9}$ Centre $G$ Deshayes, Auray, France; ${ }^{10}$ Laboratoire de génétique moléculaire, CHU, Brest, France; ${ }^{11}$ Service de génétique, Hôpital Charles Nicolle, Rouen, France; ${ }^{12}$ Service d'ORL, Hôpital Charles Nicolle, Rouen, France; ${ }^{13}$ Unité de génétique médicale, Hôpital d'Enfants Armand-Trousseau, AP-HP, Paris, France

Mutations in GJB2 are the most common cause of congenital nonsyndromic hearing loss. The controversial allele variant M34T has been hypothesized to cause autosomal dominant or recessive nonsyndromic hearing impairment and some in vitro data has been consistent with this hypothesis. In this report, we present the clinical and genotypic study of $\mathbf{1 1}$ families (seven familial forms of nonsyndromic sensorineural hearing loss (NSSNHL) and four sporadic cases) in which the M34T GJB2 variant has been identified. The M34T mutation did not segregate with the deafness in six of the seven familial forms of NSSNH. Eight persons with normal audiogram presented a heterozygous M34T variation and five normal hearing individuals were composite heterozygous for M34T and another GJB2 mutation. Four normal hearing individuals with a documented audiogram were M34T/35delG and one was M34T/(GJB6-D13S1830)del. Screening a French control population of 116 subjects we have found an M34T allele frequency of $1.72 \%$. This percentage was not significatively different from the prevalence of the M34T allele in the deaf population, which was $2.12 \%$. All these data suggest that the M34T variant is not clinically significant in human and is a frequent polymorphism in France.

European Journal of Human Genetics (2004) 12, 279-284. doi:10.1038/sj.ejhg.5201147

Published online 24 December 2003

Keywords: M34T variant; connexin 26 gene; GJB2

*Correspondence: Dr S Marlin, Unité de génétique médicale, Hôpital d'Enfants Armand-Trousseau, AP-HP, 26 av du Dr Arnold Netter, 75012 Paris, France. Tel: +33 14473 6727; Fax: +3314473 6964;

E-mail: sandrine.marlin@trs.ap-hop-paris.fr

Received 8 April 2003; revised 22 October 2003; accepted 23 October 2003
Introduction

Hearing loss is the most frequent of the sensory defects. One child in 1000 at birth presents with a severe or a profound deafness and one of 1000 more becomes deaf before adulthood. Recent discoveries in human genetics indicate that more than $80 \%$ of congenital deafness is of genetic origin in developed countries. ${ }^{1}$ The vast majority, 
80-90\%, of the nonsyndromic sensorineural hearing loss (NSSNHL) shows autosomal recessive transmission. Since 1994, more than 60 loci of NSSNHL have been identified and 28 different genes cloned. ${ }^{1}$ One of them, GJB2, encoding connexin 26 is involved in the most frequent form of NSSNHL: DFNB1 (MIM 220290) on chromosome 13p12. ${ }^{2}$ This gene has also been implicated in an autosomal dominant form of NSSNHL and in syndromic deafness. ${ }^{3,4}$

More than 60 different GJB2 amino-acid substitutions have been reported to date as recessive (DFNB1) and dominant (DFNA3) (MIM 6011544) hearing loss alleles (see The Connexin-Deafness Homepage). One of these, M34T is a missense mutation affecting a methionine in the first transmembrane domain of GJB2, conserved in GJB1 and GJB2 but not in other $\beta$-connexins. To date the effect of the M34T mutation remains controversial. The M34T mutation was first detected in a dominant pedigree of NSSNHL. ${ }^{5}$ This observation was consistent with the results of the functional data using Xenopus oocytes, which show a dominant negative effect of the M34T mutant polypeptide. ${ }^{6}$ Further studies on the first family described by Kelsell have shown dermatological signs in deaf patients and isolated another dominant mutation in GJB2 segregating with the disease. ${ }^{7}$ Several authors described normal hearing in M34T heterozygous carrier. ${ }^{8-10}$ The M34T mutation was also reported to be responsible for a recessive form of NSSNHL. ${ }^{11-13}$ In vitro data in mammalian Hela cells seem to demonstrate that M34T could disrupt the normal channel formation or oligomerisation. ${ }^{14-16}$ However, others and we have reported normal hearing subjects carrying a compound heterozygous mutation:M34T/ 35delG. ${ }^{10,17}$ Furthermore, Cucci et al ${ }^{18}$ argue that the M34T allele variant may be dependent on the mutations segregating in the opposite allele. The M34T substitution was also implicated in the phenotypic expression of association of a palmoplantar keratoderma (PPK) with deafness $^{7}$ (MIM 124500).

In this study, we have screened affected patients and normal hearing controls from France for the M34T mutation. These analyses were performed in order to determine the pathogenic effect of the M34T variant and also to assess the frequency of this variant in the French population.

\section{Materials and methods Patients}

We enrolled 188 unrelated French families that had members presented with a phenotype compatible with DFNB1 (NSSNHL bilateral, prelingual, with no cochleovestibular malformation, 110 sporadic and 88 autosomal recessive) and 116 normal hearing controls. Every control subject had normal audiometry and was questioned to eliminate familial history of deafness. In each deaf patient, a complete medical history was obtained to determine the age of onset of the deafness and to exclude the possibility of environmental causes. The deaf subjects underwent an otoscopic examination in an ear nose and throat clinic and a general examination with systematic search for signs suggestive of a syndromic form of deafness in a genetic clinic. They also had an ophtalmologic evaluation, investigation for hematuria and proteinuria and an electrocardiogram. High-resolution temporal bone computed tomography was performed. Controls, deaf children and their parents underwent pure-tone audiometry with a diagnostic audiometer in a soundproof room, with recording of pure-tone air- and bone-conduction thresholds. Airconduction pure-one average (ACPTA) thresholds in the conversational frequencies $(0.5,1,2$ and $4 \mathrm{kHz})$ were calculated for each deaf ear and were used to define the severity of deafness. The protocol was accepted by the Committee for the Protection of Individuals in Biochemical Research as required by French legislation and informed consent was obtained from all patients or their parents.

\section{Genetic analysis}

Blood samples were obtained from one or more affected patient in each family and from the controls. Genomic DNA was isolated from whole blood using Quiamp DNA Blood kits (Quiagen S.A).

Patients were tested for GJB2 mutations, by denaturing gel gradient electrophoresis analysis (DGGE) and direct sequencing of the coding exon, as described in detail elsewhere. ${ }^{2,19}$ When a monoallelic mutation of GJB2 was found, a mutation in the first exon of GJB2 was searched by direct sequencing. A specific PCR assay for the GJB6D13S1830 deletion using the method described by Del Castillo was performed when no mutation or a monoallelic mutation was identified in the GJB2. ${ }^{20}$ When the PCR was positive, the GJB6-D13S1830 deletion was confirmed by sequencing. ${ }^{2,19}$

\section{Results \\ Detection of M34T variant in deaf patients}

A total of 79 (45 of the sporadic group, 34 of the autosomal recessive group) of the 188 unrelated French families, which had members presented with a phenotype compatible with DFNB1, had a GJB2 mutation. In seven families (Figure 1, families no. 1-no. 7), one or more deaf patients had the M34T variant. In five of them (families no. 1-no. 4 and family no. 6), the M34T mutation was heterozygous. The M34T variation was homozygous in the two deaf brothers (family no. 7). In family no. 5, a sporadic case carried a composite heterozygous mutation : M34T/H100Y. In four families (family nos. 8, 9, 10), the variant M34T was detected only in hearing relatives of the hearing-impaired patients (four parents and one sister). 

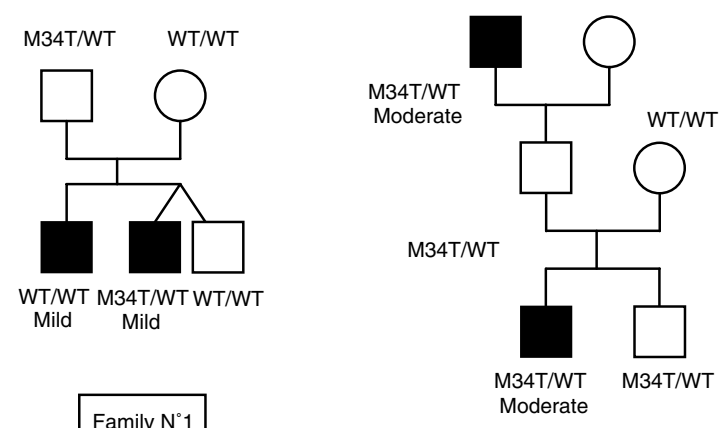

Family $\mathrm{N}^{\circ} 1$

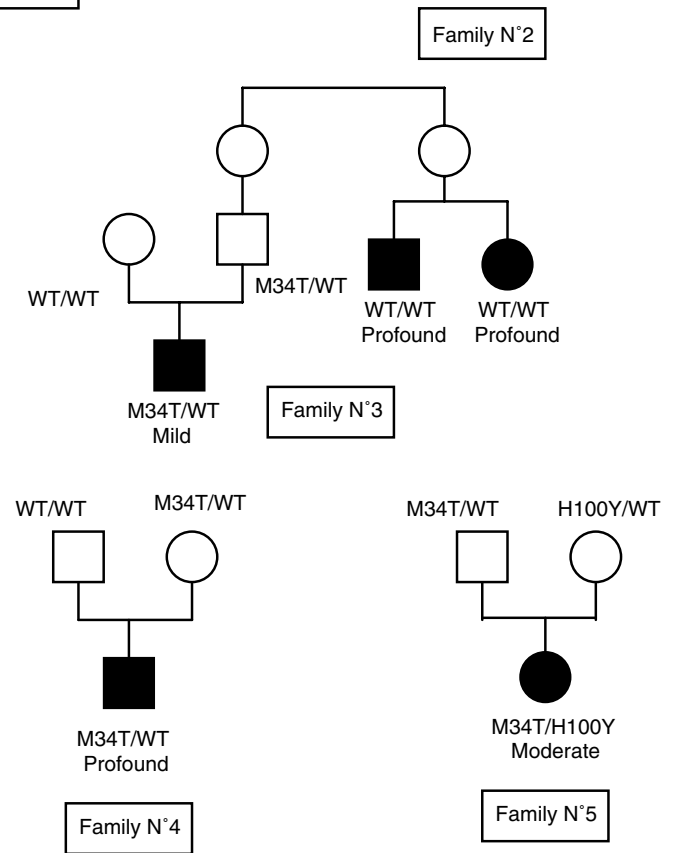

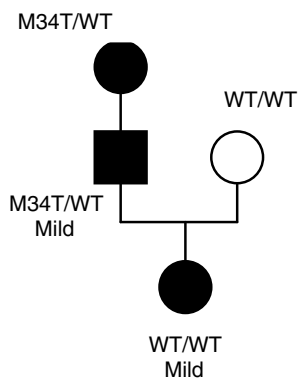

Family $\mathrm{N}^{\circ} 6$
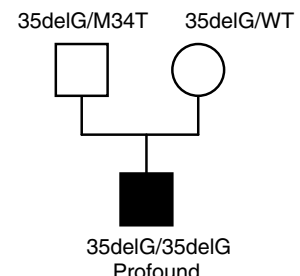

Family $\mathrm{N}^{\circ} 8$

M34T/delGJB6 WT/35delG

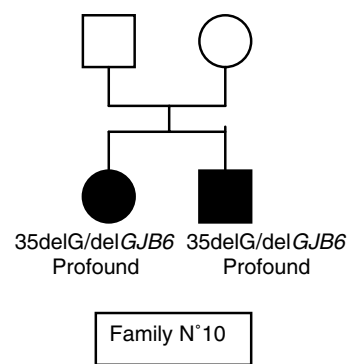

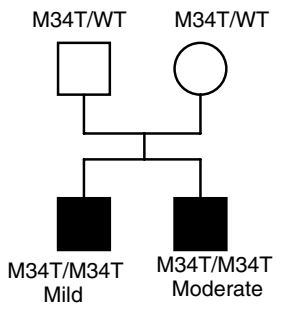

Family $\mathrm{N}^{\circ} 7$

35delG/WT M34T/35delG

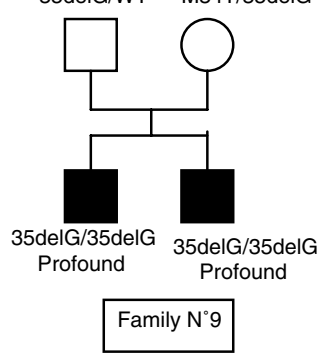

M34T/35delG

WT/35delG

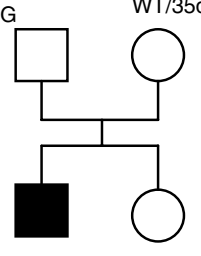

35delG/35delG M34T/35delG Profound

Family $\mathrm{N}^{\circ} 11$

Figure 1 CX26 sequence variants in the 11 families. Hearing-impaired individuals are indicated by solid symbols, unaffected patients are symbolized with open symbols. The severity of the hearing impairment is indicated.

The M34T mutation did segregate with the deafness in only one (family no. 7) of the seven familial forms of NSSNHL (Figure 1). Eight persons with normal audiogram (seven parents and one brother of a deaf child) had a heterozygous M34T variation (Figure 2). A composite heterozygous mutation (M34T/35delG or M34T/(GJB6D13S1830)del) was found in five normal hearing individuals (four parents and one sister of a deaf child) (Figures 1 and 2). In three pedigrees (families nos. 1, 3, 6), the genotype was not identical in the hearing-impaired relatives of the same family (Figure 1 ).

\section{Detection of M34T variant in control patients}

A heterozygous M34T mutation was identified in four of the 116 unrelated control patients showing a normal audiogram without any family history of deafness. The frequency of the M34T heterozygous hearing patients was
$3.44 \%(4 / 116$ cases) and the frequency of the mutated allele in the French population was $1.72 \%$ (4/232 alleles). These percentages are not significantly different from the frequency of M34T variant in the deaf population $(3.72 \%$ or $7 / 188$ families versus $4 / 116, P>0.05)$ or from the frequency of the allele in the hearing-impaired population $(2.12 \%$ (8/376) alleles versus $1.72 \%(4 / 232)$, $P>0.05)$.

\section{Discussion}

To date more than 60 GJB2 mutations have been described in deaf patients in the literature. If the pathogenycity of the truncating mutations is highly probable, the pathogenicity of missense mutations is more difficult to determine. It is difficult to differentiate a frequent polymorphism from a pathogenic mutation. We reported here 


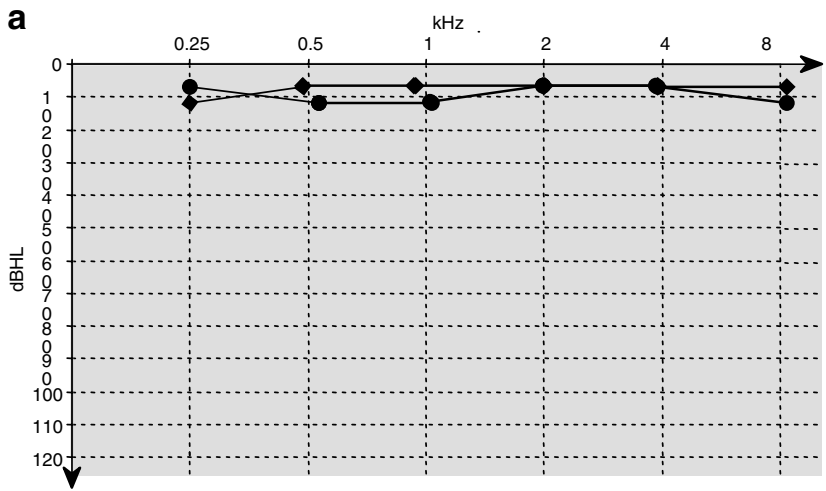

b

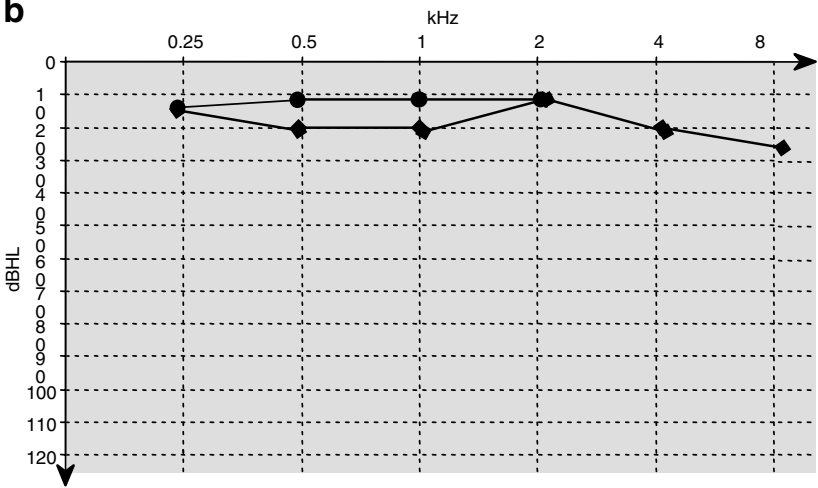

C

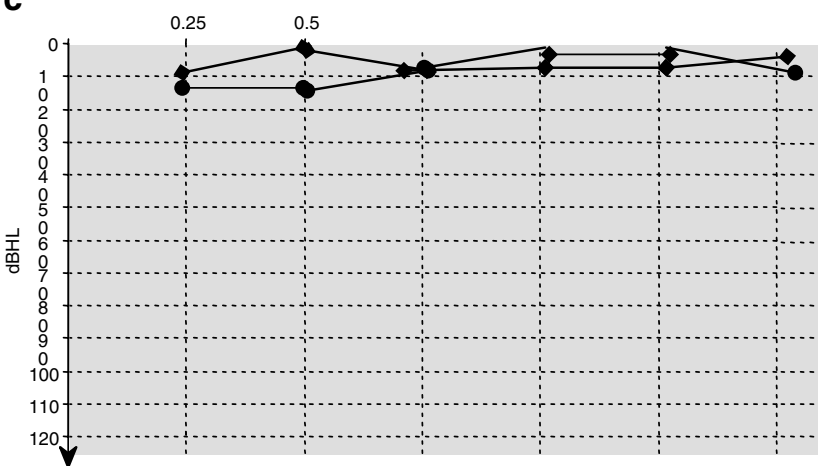

e
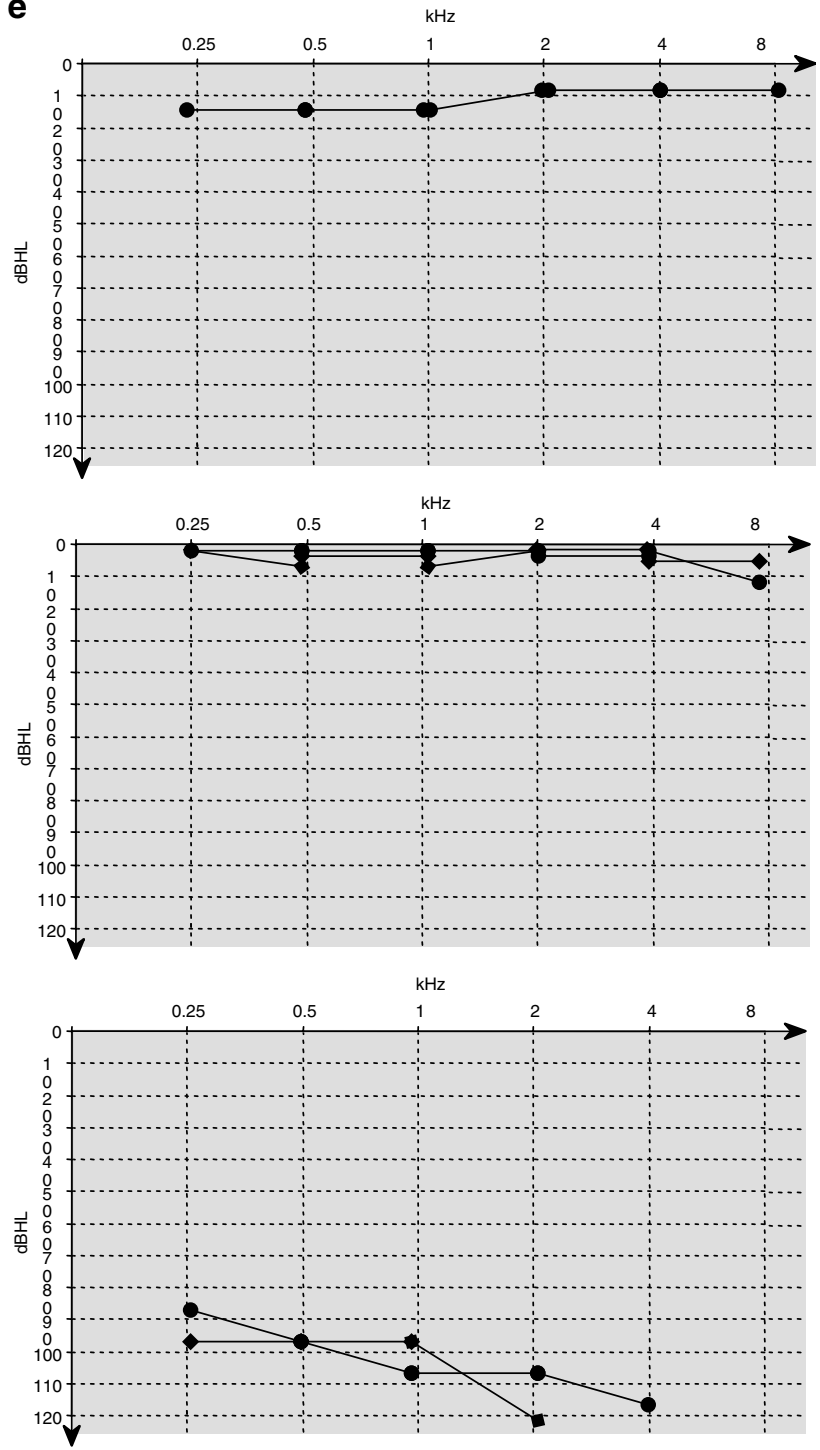

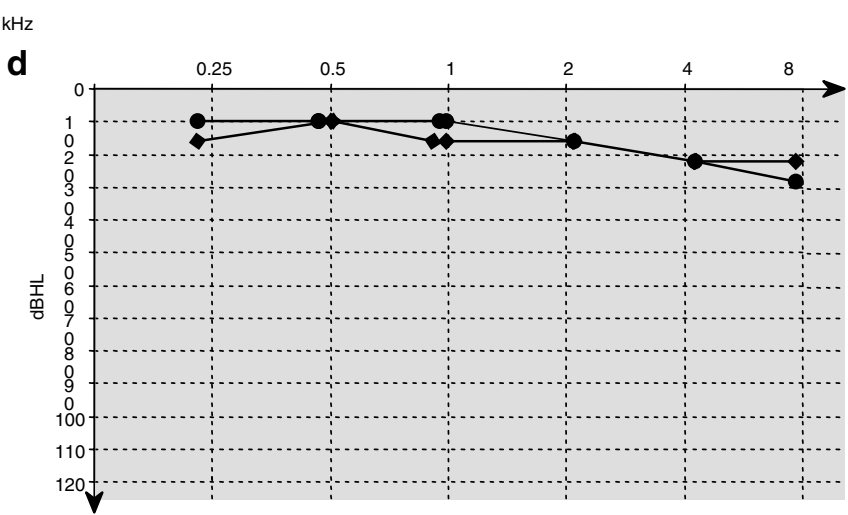

Figure 2 Audiometric curves of (a) the hearing father of family no. 2 (M34T/WT); (B) the hearing father of family no. 8 (35delG/M34T); (C) the hearing mother in family no. 9 ((35delG/M34T); (D) the hearing father in family no. 10 (M34T/ delGJB6). (E) In family no. 11: 1. the hearing father (35delG/M34T) (air-conduction thresholds egal in both ears), 2. the hearing sister (35delG/M34T), 3. The deaf brother (35delG/35delG). The circles represent the air-conduction thresholds of the right ear and the lozenges the air-conduction thresholds of the left ear. 
evidence for the nonpathogenic character of the M34T variant and its high frequency in the normal hearing population.

M34T variant was first described as an autosomal dominant mutation, ${ }^{5}$ based on its identification in patients affected both with hearing loss and PPK in a family segregating both diseases. Later, Kelsell et $\mathrm{al}^{7}$ identified in the same family a heterozygote $\mathrm{D} 66 \mathrm{H}$ mutation in GJB2 in trans of the M34T mutation and a heterozygote mutation R32W in GJB3. Later, the $\mathrm{D} 66 \mathrm{H}$ was demonstrated to be responsible for PPK and hearing defect. $^{21}$ On the other hand, Kellsell et al ${ }^{7}$ proposed that the M34T variant of GJB2 and the R32W mutation of GJB3 may contribute to the severity of the skin and hearing diseases. However, the R32W variant of GJB3 has been demonstrated not to be the cause of deafness or skin disease in Spanish patients, even in association with GJB2 mutations. ${ }^{22}$ In his report, Kelsell et al did not consider that the difference of the severity of the hearing defect could be age dependent as in an other family also carrying $\mathrm{D} 66 \mathrm{H}^{21}$ in which the adults members suffered from moderate to severe hearing loss and the children were only mildly affected. The pedigree of Kelsell's family is consistent with this hypothesis because the younger patient (III3) has PPK without deafness. The expression analysis in Hela cells ${ }^{14,15}$ suggested a functional role for M34T as a dominant allele. However, the normal audition of heterozygous M34T carriers, in the general population and in the siblings of deaf patients, like our series and in other reports, ${ }^{8-10}$ indicate that this variation is not sufficient to cause a dominant deafness in these patients. D'Andrea et $a l^{15}$ argue that these clinical observations could be explained by the presence of a second site mutation that compensates the effects of the M34T variant. This hypothesis could not explain the difference of genotypes observed in the patients of two families reported here, in which the hearing loss is transmitted with an autosomal dominant mode (families no. 2 and 6). In these two families, the most probable hypothesis is that GJB2 is not involved in the phenotype. We cannot exclude a transmission of an unknown mutation of GJB2 acting with a recessive mode in family no 2 without DFNB1 linkage analysis, but it would imply that two different GJB2 undetected mutations are present in the deaf child and grandfather.

An autosomal recessive role has also been attributed to the M34T variant, because of its occurrence with other GJB2 mutations or as homozygous in deaf individuals. $^{12,11,18,10,23,13,24}$ However, as Griffith et al ${ }^{10}$ we reported here five normal hearing subjects carrying a compound heterozygous mutation in which the M34T substitution is associated in trans with a truncated mutation : the 35delG GJB2 mutation or the (GJB6D13S1830)del. ${ }^{20}$ These results indicate that M34T is not a recessive mutation responsible for hearing loss. Cucci et al ${ }^{18}$ proposed that the effect of the M34T allele may be dependent on the mutation present in trans. However, his hypothesis could not explain the absence of hearing defect in the case of a segregation with frameshift mutations. Indeed, we presented here four normal hearing individuals with M34/35delG and for the first time a normal hearing person with M34T/(GJB6-D13S1830)del. Moreover, Cucci report two brothers presenting with the same hearing defect with different genotypes: two siblings carrying the same genotype, but with defects of different severity. ${ }^{18}$ All these observations are not consistent with a variant role of M34T according to the mutation segregating on the opposite allele. Two recent reports have found a 10 base pairs deletion in the 5'UTR of GJB2 that occurs together with the M34T mutation. ${ }^{11,24}$ The last one ${ }^{24}$ have demonstrated a linkage desequilibrum between these two variants. However, in several families reported in these two studies, ${ }^{11,24}$ neither the M34T nor the -493 del10 segregated with the deafness. These observations argue against the pathogenic character of these two variants. The presence of deaf patients homozygous for the M34T variant, as in our report (family no. 7) and in others, ${ }^{11,24}$ should not be considered as an argument for the pathogenicity of this variant because if M34T is a frequent polymorphism, it could be fortuitously present in a homozygous state in some patients.

The similar prevalence of the M34T between the deaf and the hearing population observed in this report and in other ${ }^{18}$ is in favor of the absence of pathogenic effect of this variant. The frequency of the M34T allele in the French control population reported here $(1.72 \%)$ is consistent with prevalence found in other populations: ${ }^{25} 1.5 \%$ in the USA, ${ }^{8}$ $2.4 \%$ in Belgium, ${ }^{26} 1.98 \%$ in the $\mathrm{UK}^{11} 1.33 \%$ in the Grampian region. ${ }^{27}$ In another way, this variant seems to be rare in Asia. ${ }^{28,29}$

The results of functional studies of M34T mutation in various types of cells are discordant. In Xenopus oocytes, the M34T mutant polypeptide appeared to have a dominant negative effect on the intercellular coupling. ${ }^{6}$ Expression analysis in Hela cells showed that the M34T mutant has a strong membranous localization, but it fails to assemble efficiently into hexameric gap junction hemichannels and impaired intercellular coupling. ${ }^{14,16}$ An inhibition of the wild-type GJB2 channel activity by the M34T mutant was observed by D'Andrea et al when the two proteins are coexpressed. ${ }^{15}$ However, the very recent experiments performed by Oshima et al in Hela cells showed that cells expressing M34T connexins formed normal gap junction plaques and a normal permeability in the dye transfer assays. ${ }^{30}$ As our data show that the M34T variant is not a cause of deafness in our patients, these more recent in vitro results upport our conclusion that the M34T mutation is not pathogenic. 


\section{Conclusion}

The molecular diagnosis of GJB2 abnormalities has improved genetic counseling for hearing loss. Our data seem to demonstrate that the M34T variant has no phenotypic effect in humans. These results have important implications for genetic counseling. As M34T could not be considered as responsible for the hearing defect, another mutation has to be screened in the first GJB2 exon or in GJB3 or in another gene, when this variant is associated with a GJB2 pathogenic mutation in a deaf patient.

\section{Acknowledgements}

We greatly appreciate the cooperation of the families. We thank Corinne Chauve, Catherine Magnier and Sébastien Chardenoux for excellent technical assistance. This work was supported by the Fondation pour la Recherche Médicale, The Association Française contre les Myopathies, The Institut National de la Santé et de la Recherche Médicale, and The Association pour l'Etude de la Pathologie Pédiatrique.

\section{References}

1 Petit C, Levilliers J, Marlin S et al: Hereditary hearing loss. In: Scriver CR, Beaudet AL, Sly WS, Valle D. (Eds): The metabolic and molecular bases of inherited disease. Montreal: McGraw-Hill; 2001, pp 6281-6328.

2 Denoyelle F, Marlin S, Weil D et al: Clinical features of the prevalent form of childhood deafness, DFNB1, due to a connexin26 gene defect: implications for genetic counselling. Lancet 1999; 353: $1298-1303$.

3 Denoyelle F, Lina-Granade G, Plauchu $\mathrm{H}$ et al: Connexin26 gene linked to a dominant deafness. Nature 1998; 393 (6683): 319-320.

4 Rabionet R, Gasparini P, Estivill X: Molecular genetics of hearing impairment due to mutations in gap junction genes encoding beta connexins. Hum Mutat 2000; 16 (3): 190-202.

5 Kelsell DP, Dunlop J, Stevens HP et al: Connexin 26 mutations in hereditary non-syndromic sensorineural deafness. Nature 1997; 387: $80-83$

6 White TW, Deans MR, Kelsell DP et al: Connexin mutations in deafness. Nature 1998; 394: 630-631.

7 Kelsell DP, Wilgoss AL, Richard G et al: Connexin mutations associated with palmoplantar keratoderma and profound deafness in a single family. Eur J Hum Genet 2000; 8 (6): 469-472.

8 Kelley PM, Harris DJ, Comer BC et al: Novel mutations in the connexin 26 gene (GJB2) that cause autosomal recessive (DFNB1) hearing loss. Am J Hum Genet 1998; 62: 792-799.

9 Scott DA, Kraft ML, Carmi R et al: Identification of mutations in the connexin 26 gene that cause autosomal recessive nonsyndromic hearing loss. Hum Mutat 1998; 11 (5): 387-394.

10 Griffith AJ, Chowdhry AA, Kurima K et al: Autosomal recessive nonsyndromic neurosensory deafness at DFNB1 not associated with the compound-heterozygous GJB2 (Connexin 26) genotype M34T/167delT. Am J Hum Genet 2000; 67: 745 -749.

11 Houseman MJ, Ellis LA, Pagnamenta A et al: Genetic analysis of the connexin-26 M34T variant: identification of genotype M34T/
M34T segregating with mild-moderate non-syndromic sensorineural hearing loss. J Med Genet 2001; 38: 20-25.

12 Wilcox SA, Saunders K, Osborn AH et al: High frequency hearing loss correlated with mutations in the GJB2 gene. Hum Genet 2000; 106: 399-405.

$13 \mathrm{Wu} \mathrm{B}$, Lindeman $\mathrm{N}$, Lip $\mathrm{V}$ et al: Effectiveness of sequencing connexine 26 (GJB2) in cases of familial or sporadic childhood deafness referred for molecular diagnostic testing. Genet Med 2002; 4: 279-288.

14 Martin PE, Coleman SL, Casalotti SO et al: Properties of connexin26 gap junctional proteins derived from mutations associated with non-syndromal heriditary deafness. Hum Mol Genet 1999; 8: 2369-2376.

15 D'Andrea P, Veronesi V, Bicego $\mathrm{M}$ et al: Hearing loss: frequency and functional studies of the most common connexin26 alleles. Biochem Biophys Res Commun 2002; 296: 685-691.

16 Thonnissen E, Rabionet R, Arbones M et al: Hum Genet 2002; 111: 190-197.

17 Marlin S, Garabédian E-N, Roger G et al: Connexin26 gene mutations in congenitally deaf children: pitfalls for genetic counselling. Arch Otolaryngol Head Neck Surg 2001; 127: 927-933.

18 Cucci R, Prasad S, Kelley P et al: The M34T allele variant of connexin 26. Genet Test 2000; 4: 335-344.

19 Antoniadi T, Grønskov K, Sand A et al: Mutation analysis of the GJB2 (connexin 26) gene by DGGE in Greek patients with sensorineural deafness. Hum Mut 2000; 16: 381-382.

20 Del Castillo I, Villamar M, Moreno-Pelayo M et al: A deletion involving the connexine 30 gene in nonsyndromic hearing impairement. N Engl Jal of Medecine 2002; 346: 243-249.

21 Maestrini E, Korge BP, Ocana-Sierra J et al: A missense mutation in connexin 26, D66H, causes mutilating keratoderma with sensorineural deafness (Vohwinkel's syndrome) in three unrelated families. Hum Mol Genet 1999; 8: 1237-1243.

22 López-Bigas $\mathrm{N}$, Olivé $\mathrm{M}$, Rabionet $\mathrm{R}$ et al: Connexin 31 (GJB3) is expressed in the peripheral and auditory nerves and causes neuropathy and hearing impairment. Hum Mol Genet 2001; 10: 947-952.

23 Griffith AJ, Kelsell DP, Houseman MJ et al: Genetic analysis of the connexin-26 M34T variant. J Med Genet 2001; 38: e24.

24 Zoll B, Petersen L, Lange K et al: Evaluation of Cx26/GJB2 in German hearing impaired persons: mutation spectrum and detection of desiquilibrum between M34T (c.101T $>$ C) and -493del10. Hum Mutat 2003; 21: 98.

25 Kenneson A, Van Naarden Braun K, Boyle C: GJB2 (connexin 26) variants and nonsyndromic sensorineural hearing loss: a HuGE review. Genet Med 2002; 4: 258-274.

26 Hilbert P, Van Maldergem L, Gillerot Y: Use of denaturating high performance liquid chromatography (DHPLC) for connexin 26 gene mutation screening. Am J Hum Genet 1999; 65: A301.

27 Rice J, Clark C, Fraser L et al: Connexin 26 mutation M34T is a common finding in non syndromal deaf children in the Grampian region. J Med Genet 1999; 36 (Suppl. 1): S97.

28 Abe S, Usami S-i, Shinkawa $\mathrm{H}$ et al: Prevalent connexin 26 gene (GJB2) mutations in Japanese. J Med Genet 2000; 37: 41-43.

29 Park HJ, Hahn SH, Chun YM et al: Connexin26 mutations associated with nonsyndromic hearing loss. Laryngoscope 2000; 110: $1535-1538$

30 Oshima A, Doi T, Mitsuoka K et al: Roles of M34, C64, and R75 in the assembly of human connexin 26: implications for key amino acid residues for channel formation and function. J Biol Chem 2002; 278: 1807-1816. 